\title{
latrogenic Mitral Valve Perforation following CABG and Aortic Valve Replacement: A Rare Complication detected by Post-bypass Transesophageal Echocardiography
}

\author{
${ }^{1}$ Ravi Raj, ${ }^{2}$ Sarin Mathew, ${ }^{3}$ Goverdhan Dutt Puri
}

\begin{abstract}
latrogenic mitral valve perforation following aortic valve replacement is a rare complication. We present a case of anterior mitral leaflet perforation following coronary artery bypass grafting (CABG) and aortic valve replacement detected by intraoperative transesophageal echocardiography (TEE). A 57-year-old male patient with preoperative diagnosis of coronary artery disease, sclerodegenerative aortic valve with severe aortic stenosis and mild central mitral regurgitation (MR) underwent CABG and aortic valve replacement. A post bypass TEE in midesophageal long axis view showed an additional MR jet across the body of the anterior mitral leaflet. We present intraoperative TEE images with a discussion on role of TEE in detection of mitral valve perforation and surgical decision making.
\end{abstract}

Keywords: Iatrogenic, Mitral valve perforation, Aortic valve replacement, Coronary artery bypass grafting, Transesophageal echocardiography.

Abbreviations: AVR: Aortic valve replacement; CABG: Coronary artery bypass grafting; 2D: Two-dimensional; MR: Mitral regurgitation; TEE: Transesophageal echocardiography; TTE: Transthoracic echocardiography.

How to cite this article: Raj R, Mathew S, Puri GD. latrogenic Mitral Valve Perforation following CABG and Aortic Valve Replacement: A Rare Complication detected by Post-bypass Transesophageal Echocardiography. J Perioper Echocardiogr 2014;2(2):75-76.

Source of support: Nil

Conflict of interest: None declared

\section{INTRODUCTION}

Mitral valve leaflet perforation is most commonly due to infective endocarditis. ${ }^{1}$ Other causes can be due to

\footnotetext{
${ }^{1}$ Senior Resident, ${ }^{2}$ Assistant Professor, ${ }^{3}$ Professor

1,3 Department of Anesthesia and Intensive Care, Postgraduate Institute of Medical Education and Research, Chandigarh, India

2Department of Cardiothoracic and Vascular Surgery, Postgraduate Institute of Medical Education and Research Chandigarh, India

Corresponding Author: Ravi Raj, Senior Resident Department of Anesthesia and Intensive Care, Postgraduate Institute of Medical Education and Research, Chandigarh, India e-mail: drraviraj2007@gmail.com
}

autoimmune disease like systemic lupus erythematosus or antiphospholipid syndrome. ${ }^{2}$ Iatrogenic mitral valve perforation is a rare complication following aortic valve replacement. We report a case of anterior mitral leaflet perforation following coronary artery bypass grafting (CABG) and aortic valve replacement.

\section{CASE HISTORY}

A 57-year-old male patient presented with complaints of progressive dyspnea on exertion and occasional palpitations. A preoperative diagnosis of severe aortic stenosis, sclerodegenerative aortic valve with normal left ventricular function was made on transthoracic echocardiography. A coronary angiogram in same patient showed single vessel disease involving proximal left anterior descending artery causing $80 \%$ stenosis. The patient was scheduled for aortic valve replacement (AVR) and CABG. Inside the operating room, after induction of anesthesia, a 2D TEE probe (S7-2 Omni; Philips IE33, Andover, MA, USA) was inserted which showed a thick, calcified bicuspid aortic valve with severe aortic stenosis and normal left ventricular systolic function. Mitral valve was morphologically normal with a mild central mitral regurgitation jet (vena contracta: $0.287 \mathrm{~cm}$; Fig. 1). Patient underwent CABG and AVR (19 mm Trifecta; bioprosthetic) under cardiopulmonary bypass support.

Post CPB TEE examination showed 2 mitral regurgitation jets in midesophageal long axis view (Fig. 2). There was a mild central MR jet and an additional mild MR jet across the body of the anterior mitral leaflet with vena contracta of $0.312 \mathrm{~cm}$. Transgastric short axis view showed turbulence in the anterior leaflet in the region of the A1 scallop. Other findings included normal functioning prosthetic aortic valve and normal left ventricular systolic function. The findings of post bypass TEE were conveyed to operating team and a decision was taken to not to intervene for mitral perforation considering mild nature of mitral regurgitation. The vena contracta measured for MR jet at suspected perforation site reduced to $0.26 \mathrm{~cm}$ after protamine administration. The postoperative course was uneventful and a repeat transthoracic echocardiography before discharge showed only trace MR. 


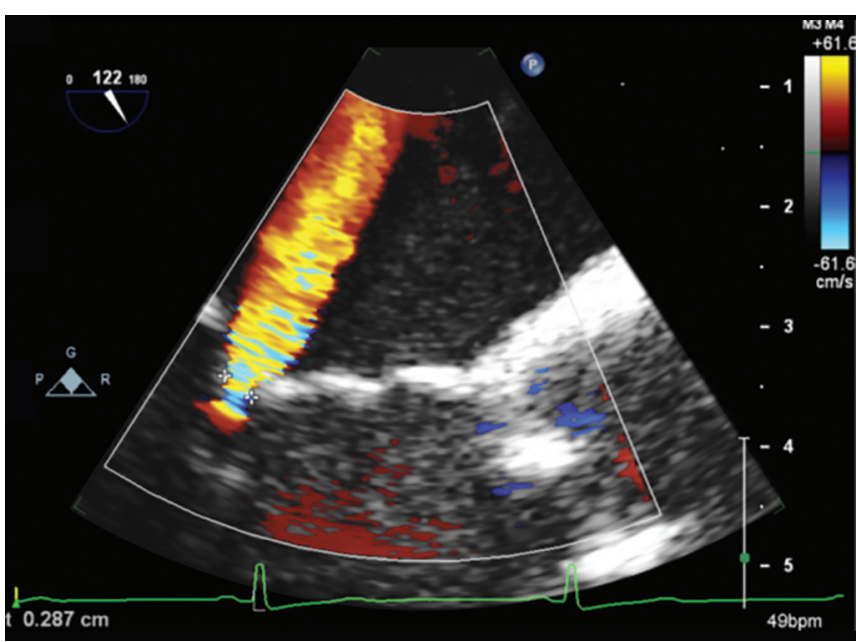

Fig. 1: Midesophageal long-axis view showing mild central mitral regurgitation in pre CPB period

\section{DISCUSSION}

Iatrogenic mitral valve regurgitation following AVR is a rare complication. Dyck et al in their review of complications in 475 cases following repair for aortic valve insufficiency reported two cases of perforation of base of anterior mitral leaflet. ${ }^{3}$ The anterior mitral leaflet is in fibrous continuity with the aortic valve through the aortomitral annulus/curtain. The commissure between the left and noncoronary sinuses of the aortic valve is located over the middle of the anterior mitral leaflet. Due to this close anatomical proximity, either of the 2 valves may be injured during intervention on the other.

Similarly, there are a few case reports on aortic valve perforation following mitral valve repair/replacement. ${ }^{4-6}$ Injury to noncoronary cusp is the most common mechanism for aortic valve perforation during mitral valve surgery but may involve left coronary cusp also.

In our case, aortic valve was bicuspid, thick and calcified. Intraoperatively, specks of calcification were found extending upto anterior mitral leaflet by the surgical team. The mechanism of mitral valve perforation in our case could be due to avulsion of calcified specks on anterior mitral leaflet while removing heavily calcified native aortic valve. Intraoperative TEE was instrumental in detection of the suspected anterior mitral leaflet perforation and assessment of the resulting MR severity. The decision to not to intervene was taken considering mild MR at the suspected perforation site and the measured vena contracta reduced further after protamine administration.

Transesophageal echocardiography is the imaging modality of choice for assessing mitral valve perforation and resulting mitral regurgitation. Color Doppler jet area method for estimation can lead to overestimation of MR severity in presence of multiple MR jets due to increased

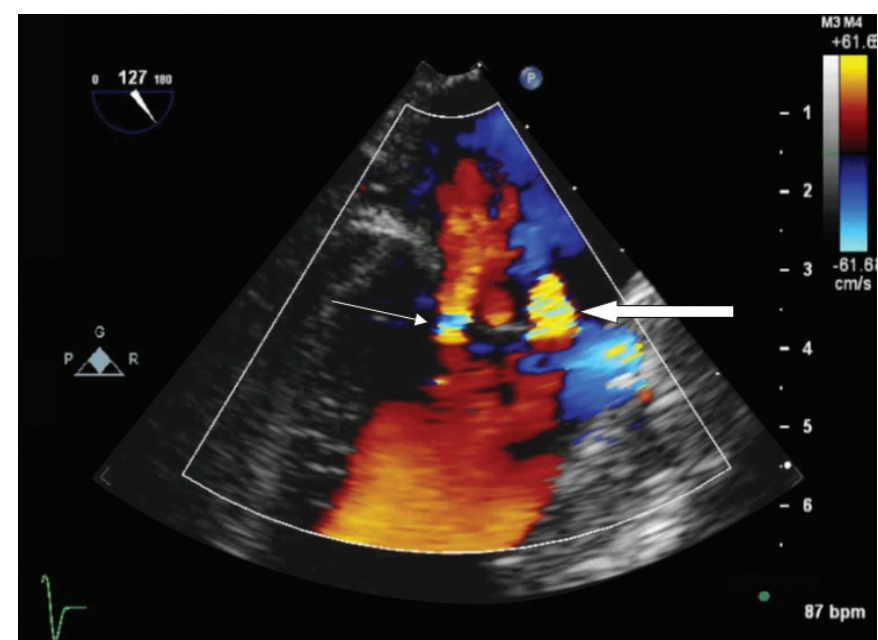

Fig. 2: Midesophageal long-axis view showing 2 jets of mitral regurgitation (MR) in post CPB period. Please note mild central MR (thin arrow) and another jet of MR (thick arrow) across the body of the anterior mitral leaflet at site of suspected perforation

ambient fluid entrainment from adjacent regurgitant jets.? Alternatively, calculation of total regurgitant volume and regurgitant fraction is more accurate method. ${ }^{8} 3 \mathrm{D}$ TEE if available can provide more anatomical details regarding location and severity of mitral perforation.

\section{CONCLUSION}

We report a rare case of anterior mitral leaflet perforation following aortic valve replacement in a patient with CAD and bicuspid aortic valve. Intraoperative TEE helped in detecting mitral valve perforation as well as in assessment of resulting MR severity.

\section{REFERENCES}

1. Stout KK, Verrier ED. Valvular heart disease: Changing concepts in disease management; Acute valvular regurgitation. Circulation 2009;119(25):3232-3241.

2. Bouma W, Klinkenberg T, Horst I, et al. Mitral valve surgery for Mitral regurgitation caused by Libman Sacks endocarditis: a report of four cases and a systematic review of the literature. J Cardiothoracic Surg 2010;5(13):1-13.

3. Dyck MV, Glineur D, Kerchove LD, et al. Complications after aortic valve repair and valve sparing procedures. Annals Cardiothoracic Surg 2013;2(1):130-139.

4. Hill AC, Bansal RC, Razzouk AJ, et al. Echocardiographic recognition of iatrogenic aortic valve leaflet perforation. Annals Thoracic Surg 1997;64(3):684-689.

5. Ducharme A, Courval JF, Dore A, et al. Severe aortic regurgitation immediately after mitral valve annuloplasty. Annals Thoracic Surg 1999;67(5):1487-1489.

6. Aboelnasr M, Rohn V. Aortic valve leaflet perforation after mitral valve repair. Prague Medical Report 2013;114(3):172-176.

7. Lin BA, Forouhar AS, Pahlevan NM, et al. Color Doppler jet area overestimates regurgitant volume when multiple jets are present. J Am Society of Echocardiography 2010;23(9):993-1000.

8. Zoghbi WA, Sarano ME, Foster E, et al. Recommendations for evaluation of the severity of native valvular regurgitation with two-dimensional and Doppler echocardiography. J Am Society Echocardiogr 2003;16(7):777-802. 